Dataset Paper

\title{
Efficacy and Safety of Botulinum Toxin A for Treating Bladder Hyperactivity in Children and Adolescents with Neuropathic Bladder Secondary to Myelomeningocele
}

\author{
Antonio Marte, Micaela Borrelli, Maurizio Prezioso, Lucia Pintozzi, and Pio Parmeggiani \\ Division of Pediatric Surgery, Second University of Naples, Via S. Pansini 5, 80131 Naples, Italy \\ Correspondence should be addressed to Antonio Marte; antonio.marte@unina2.it
}

Received 4 October 2012; Accepted 6 November 2012

Academic Editors: P. Crispen, T. Efferth, P. Ferrante, and B. Weinstock-Guttman

Copyright (C) 2013 Antonio Marte et al. This is an open access article distributed under the Creative Commons Attribution License, which permits unrestricted use, distribution, and reproduction in any medium, provided the original work is properly cited.

\begin{abstract}
We verified the efficacy and safety of botulinum toxin A (BTX-A) in treating bladder overactivity in children with neurogenic bladder (NB) secondary to myelomeningocele (MMC). Forty-seven patients (22, females; 25, males; age range, 5-17 years; mean age, 10.7 years) with poorly compliant/overactive neurogenic bladder on clean intermittent catheterization (CIC) and resistance or noncompliant to anticholinergics were injected with $200 \mathrm{IU}$ of BTX-A intradetrusor. All patients experienced a significant $66.45 \%$ average increase of leak point volume (Wilcoxon paired rank test $=7.169 \mathrm{e}-10$ ) and a significant $118.57 \%$ average increase of specific bladder capacity at $20 \mathrm{~cm} \mathrm{H}_{2} \mathrm{O}$ (Wilcoxon paired rank test $=2.466 \mathrm{e}-12$ ). Ten patients who presented with concomitant uni/bilateral grade II-IV vesicoureteral reflux were treated at the same time with Deflux. No patient presented with major perioperative or postoperative problems. Twenty-two patients needed a second and 18 a third injection of BTX-A after 6-9 months for the reappearance of symptoms. After a mean follow-up of 5.7 years, 38 out of 47 patients achieved dryness between CICs, and 9 patients improved their incontinence but still need pads. Our conclusion is that BTX-A represents a viable alternative to more invasive procedure in treatment of overactive NB secondary to MMC.
\end{abstract}

\section{Introduction}

Current treatment of patients with neurogenic bladder (NB) secondary to myelomeningocele (MMC) is mainly based on the clean intermittent catheterization (CIC) and the associated anticholinergic agents, or on surgical procedures on the bladder or bladder neck. In cases where the NB is characterized by overactivity or low compliance, the first safeguard is the use of early anticholinergic drugs, such as oxybutynin, tolterodine, or most recently trospium chloride with the ultimate goal of making the patient dry safeguarding renal function. If anticholinergics and CIC do not provide the desired result, then it is necessary to use more invasive techniques of expansion in order to transform the bladder into a reservoir with high capacity or low pressure [1]. On the basis of recent research, there is an increasingly assertion of the use of botulinum toxin A (BTX-A) in the treatment of NB secondary to MMC as a valid alternative to invasive procedures [2-5]. We present our experience in a selected group of patients in whom the clinical evaluation and urodynamics assumed the need for action to expand their bladder due to the poor response to drugs, and in whom incontinence and the use of pads between the catheterizations strongly influence their social lives.

\section{Methodology}

From January 2002 to June 2011, 47 patients out of 68 with neuropathic bladders were selected for BTX-A treatment. There were 22 females and 25 males (age range: 5-17 years; mean age: 10.7 years) with poorly compliant/overactive bladders on clean intermittent catheterization (CIC) and resistance or noncompliant to pharmacological therapy. Ten patients presented with concomitant uni/bilateral grade II-IV vesicoureteral reflux (VUR). Despite the treatment, the patients complained of urinary incontinence between catheterizations. 


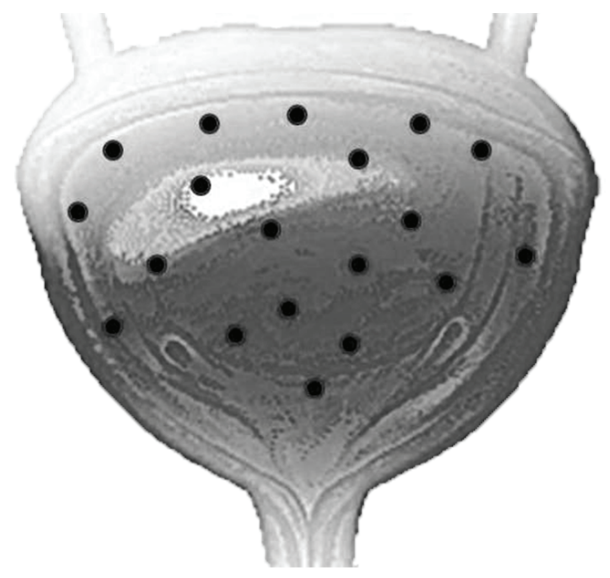

FIGURE 1: Multipoint injection scheme $\left(1 \mathrm{~cm}^{3}=10 \mathrm{IU}\right.$ of BTX-A injected in 20 sites, sparing periureteral areas).

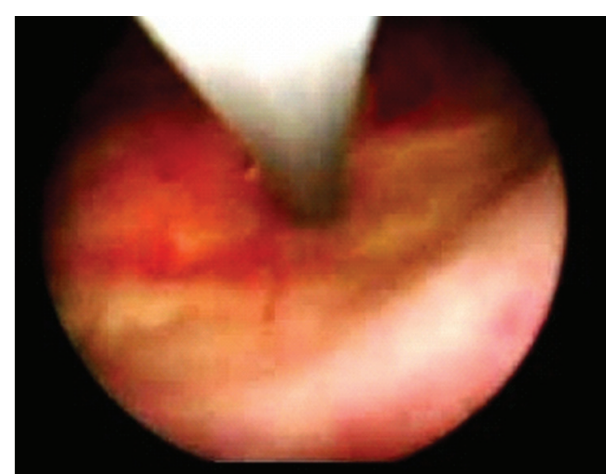

FIgURE 2: Injection of the toxin in the detrusor. The submucosal protrusion shows that the toxin has been properly injected.

All patients were regularly monitored with periodic urodynamic examinations, voiding cystourethrography, and prophylactic antibiotics. As an imperative condition, there was the requirement that all the patients had negative urine cultures prior to the procedure. The solution used for injection was obtained by preparing 2 vials of BTX-A diluted in $20 \mathrm{~cm}^{3}$ of $0.9 \%$ saline solution for a total of $200 \mathrm{IU}$, avoiding shaking the vial too roughly. The needle was introduced for 4 $5 \mathrm{~mm}$ into the bladder wall. The injection was performed by a needle-metallic cannula of $3.7 \mathrm{Ch}$ with $23 \mathrm{G}$ needle of the type used for the correction of reflux. The needle was introduced for 4-5 mm of its entire length in the bladder wall, and in each site, a quantity of BTX-A of about $1 \mathrm{~cm}^{3}$ was introduced for a total of 20 injections.

With patients in the lithotomy position, the injection was carried out with the bladder being filled to about $20 \%$ to $30 \%$ of its expected capacity. The administration of toxin was carried out in general or local anesthesia in more collaborative patients: in the latter 15 minutes prior to the procedure, $15-25 \mathrm{~cm}^{3}$ of lidocaine to $0.5 \%$ was instilled into the bladder through the catheter. After checking the appearance of the bladder and ureteral ostia, then BTXA was injected according to a blind multipoint procedure.

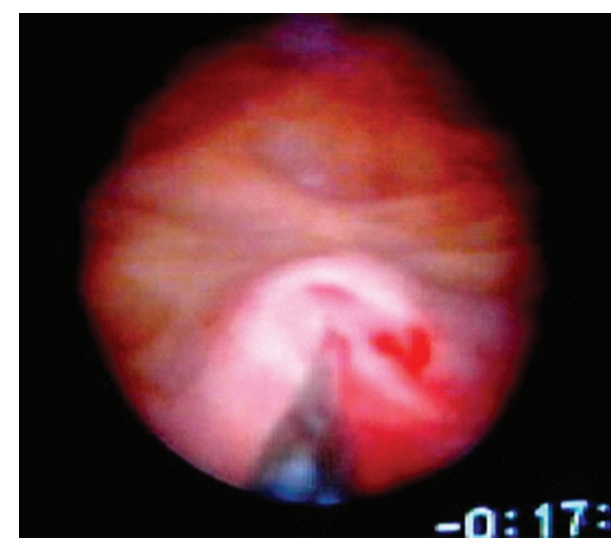

FIGURE 3: Correction of VUR in trabeculated neuropathic bladder. Notice the volcano appearance of the orifice.

Injections were performed without sparing the trigone, but avoiding periureteral areas in order not to interfere with the ureterovesical dynamics, in 20 different sites taking care not to insert the needle too deeply, especially at the level of the bladder dome for avoiding inadvertently intra-abdominal viscera injection. The appearance of a submucosal ledge was considered a correct injection. When there were trabeculations, they were not spared from the injections as they derived from a focal detrusor hypertrophy. A catheter was kept in place for 24 hours, and an antibiotic was administered for 5 days. In patients with VUR, the correction by endoscopic subureteral injection of Deflux was performed at the end of injections utilizing 1 to $3 \mathrm{~cm}^{3}$ of bulging agent realizing the so-called total endoscopic management (TEM) [6]. In these latter cases, the antibiotic prophylaxis was maintained for 1 month after the treatment (Figures 1, 2, and 3).

The follow-up with ultrasound, urodynamics, and clinical evaluation mainly for the dryness was made at 6, 12, and 24 weeks. A VCUG was performed 12 weeks after the procedure in patients with VUR, and reflux was considered cured if a complete resolution was obtained or a downgrading to the first grade. In case of persistence, the endoscopic injection was repeated. The urodynamic parameters used were leak point pressure and leak point volume (LPP and LPV) and the specific bladder capacity at $20 \mathrm{~cm} \mathrm{H}_{2} \mathrm{O}$ (SC 20). This index, similar to the compliance, provides a useful functional datum because it indicates the ability of bladder to fill at low pressure, within the limits of $20 \mathrm{~cm} \mathrm{H}_{2} \mathrm{O}[7,8]$. The values obtained were subjected to statistical Wilcoxon tests for paired ranks.

\section{Dataset Description}

The dataset associated with this Dataset Paper consists of one item, which is described as follows.

Dataset Item 1 (Table). Demographic data of patients treated with BTX-A (leak point volume, leak point pressure, and specific capacity at $20 \mathrm{~cm} \mathrm{H}_{2} \mathrm{O}$ are referred to baseline values and after the first injection of BTX-A). No patient presented 


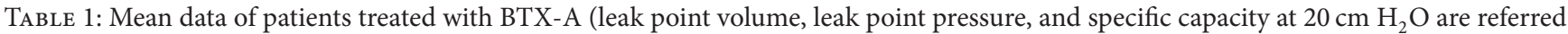
to baseline values and after the first injection of BTX-A).

\begin{tabular}{cccccccc}
\hline & Age & Pre-LPV & Post-LPV & Pre-LPP & Post-LPP & Pre-SC 20 & Post-SC 20 \\
\hline Mean & 10.70 & 124.8085 & 207.7447 & 38.17021 & 38.44681 & 69.82979 & 152.617 \\
\hline
\end{tabular}

with severe systemic complication after the injection. Thirtyeight out of forty-seven patients presented with slight hematuria for 2-3 days, 2 patients had postoperative urinary tract infection, 1 patient complained of gastric pain, treated with ranitidine, 2 patients presented with facial flushing, and 5 patients complained of mild hyposthenia of the lower limbs. All patients were hospitalized for 24 hours with catheterization and dismissed on day one. All patients experienced a significant $66.45 \%$ average increase of LPV (Wilcoxon paired rank test $=7.169$ e-10) and a significant $118.57 \%$ average increase of SC 20 (Wilcoxon paired rank test $=2.466 \mathrm{e}-12$ ). Preoperative LPP and postoperative LPP are not significant (Wilcoxon paired rank test $=0.8858)($ Table 1$)$. At VCUG follow-up control, there were two recurrences of uinlateral grade III reflux that required a second endoscopic treatment. All patients are still on CIC regimen. With the exception of patient numbers $5,6,14,18,31,21$, and 28 , which are still in clinical balance and stay dry between CICs, 22 patients received a second and 18 a third injection of BTX-A after 6-9 months for the reappearance of symptoms. After a mean follow-up of 5.7 years, 38 out of 47 patients achieved dryness between CICs, and 9 patients improved their incontinence but still need pads. Ten patients have resumed anticholinergic agents (Figures 4 and 5).

\begin{tabular}{|c|c|}
\hline Column 1: & Patient Number \\
\hline Column 2: & Sex \\
\hline Column 3: & Age (y) \\
\hline Column 4: & VUR \\
\hline Column 5: & Pre-LPV (mL) \\
\hline Column 6: & Post-LPV (mL) \\
\hline Column 7: & Pre-LPP $\left(\mathrm{cm} \mathrm{H}_{2} \mathrm{O}\right)$ \\
\hline Column 8: & Post-LPP $\left(\mathrm{cm} \mathrm{H}_{2} \mathrm{O}\right)$ \\
\hline Column 9: & Pre-SC $20(\mathrm{~mL})$ \\
\hline Column 10: & Post-SC $20(\mathrm{~mL})$ \\
\hline Column & Outcome \\
\hline
\end{tabular}

\section{Concluding Remarks}

In our experience, the detrusor injection of BTX-A was simple, effective, and only mildly invasive as it does not necessarily require narcosis; it enables also the simultaneous correction of VUR to overcome the increased risk of high intravesical pressure and recurrent UTI. This treatment can decrease the incidence of renal damage in children on whom conservative management fails to help and requires only a short hospital stay; anticholinergic drugs can generally be withdrawn. The patient in this series experienced both improved LPV and specific capacity at $20 \mathrm{~cm} \mathrm{H}_{2} \mathrm{O}$. In relation

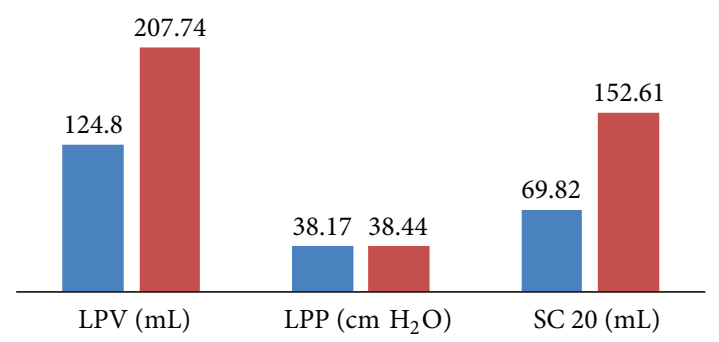

- Before

- After

Figure 4: Demographic data graph before and after the first injection of BTX-A. LPV: leak point volume; LPP: leak point pressure; SC: specific capacity at $20 \mathrm{~cm} \mathrm{H}_{2} \mathrm{O}$.

to compliance, this index is especially significant as it shows satisfactory bladder filling at low pressures.

The clinical use of BTX-A is now a common and consolidated technique in several medical fields. Urology-specifically pediatric urology-is an example of its most recent application in neuropathic and nonneuropathic patients [9]. BTX-A blocks acetylcholine release by binding, at the presynaptic level, to SNAP-25, a cytoplasmic protein on the cell membrane, which plays a major role in acetylcholine release $[10,11]$. The duration of the relaxing effect on the detrusor muscle is not yet known, although the repeated applications-aside from being well tolerated-seem to prolong both duration and efficacy of treatment. Another problem is the most suitable dosage in relation to the characteristics of the detrusor: in our patients, we used $200 \mathrm{IU}$ of BTX-A independently of age and cystometric curve. Dosage was empirically defined within a safety margin, based on other reports in the literature; therefore even if our results are satisfactory, further experience in this field will most likely help define the most appropriate toxin dosage needed [1214]. Although the small number of patients studied does not allow us to draw definite conclusions, the results show that the use of BTX-A can be suitably included in the algorithm for the treatment of neurogenic bladder and detrusor hyperreflexia, after anticholinergic drugs-or even the first treatment in the event of failure of drug treatment. The use of more invasive surgical operations, such as autoaugmentation or enterocystoplasty, could be taken into account only in case BTX-A treatment fails. The range of therapeutic options for this condition can safely include BTX-A treatment, along with anticholinergic drugs and traditional surgery. However, the duration of its efficacy and long-term reliability in urology should be further investigated and standardized, although the experience has shown that the technique can be safely readministered after 6 months with good results. Moreover, 


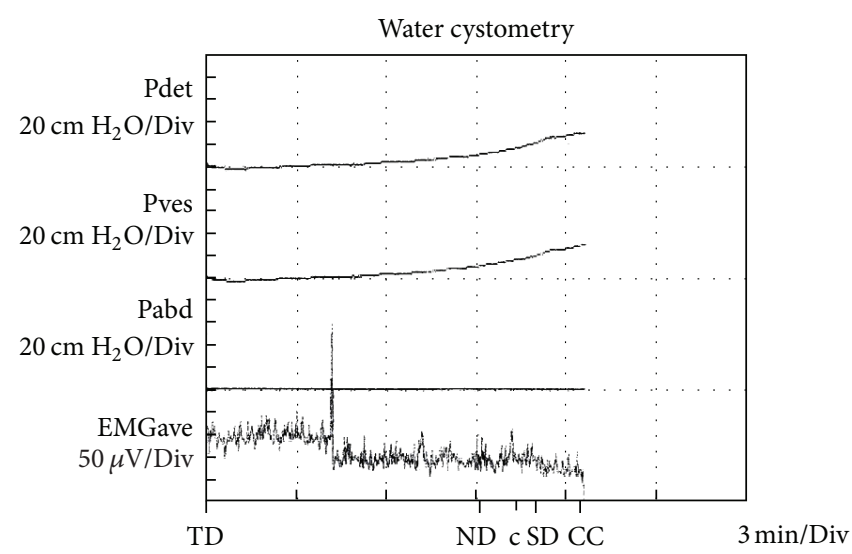

(a)

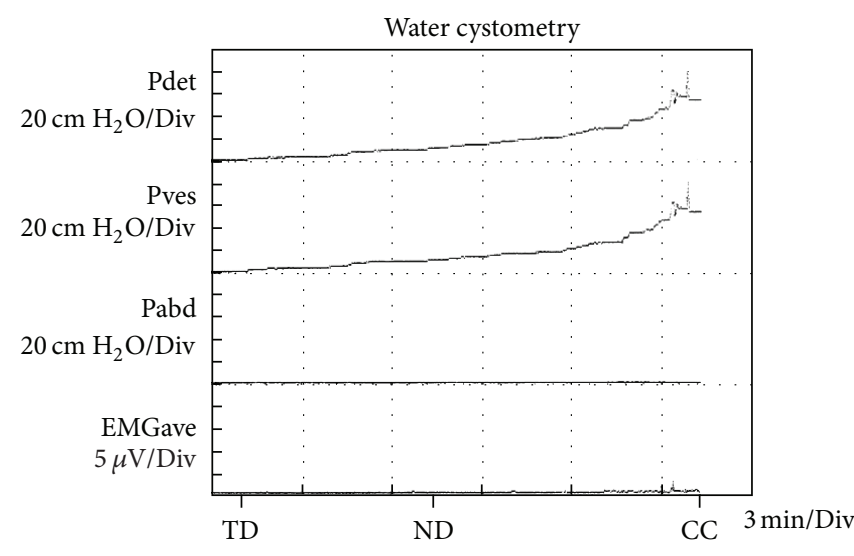

(b)

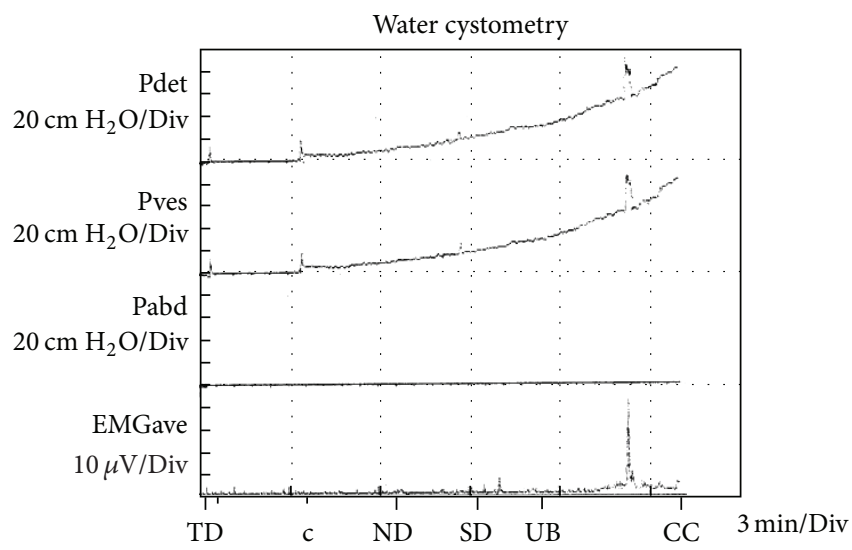

(c)

FIGURE 5: Patient number 27: evolution of the patient's urodynamics. (a) Basic urodynamics. (b) Clinical balance and good bladder capacity after the first injection of BTX-A. (c) Eight months after the bladder pressure exceeded $40 \mathrm{~cm}_{2} \mathrm{O}$ quickly (safety pressure), requiring a new treatment.

no relevant side effects were detected after the administration of BTX-A. This also means that patients require constant clinical and instrumental controls. Another recently highlighted factor concerns the depth of the injection into the bladder. As demonstrated by Mehnert et al. [15] with a study by the use of MRI with toxin labeled with gadolinium, a too deep injection is deposited outside of the bladder wall. Therefore, it can be assumed that an ineffective treatment can be linked more to improper depth of the injection rather than to the dosage of toxin.

Moreover, there remain uncertain the duration of treatment, the efficacy, and long-term reliability on the bladder. It is not to be underestimated, then, the possibility that repeated dosing of BTX-A may induce the formation of antitoxin antibodies which will void effectiveness $[16,17]$. Although this event has not yet been described in use on the urinary system, it cannot be excluded that such an event may occur.

Our conclusion is that BTX-A represents a viable alternative to more invasive procedure in treatment of $\mathrm{NB}$ secondary to MMC, with good objective and subjective results, when anticholinergic agents are not effective or when their side effects lead the patient to abandon the treatment. The procedure allows also the contemporary treatment of VUR decreasing the incidence of renal damage in children on whom conservative management fails to help. However the need for a second and a third treatment is a significant factor in the cost-benefit assessment $[18,19]$.

\section{Dataset Availability}

The dataset associated with this Dataset Paper is dedicated to the public domain using the CCO waiver and is available at http://dx.doi.org/10.1155/2013/580927/dataset.

\section{Conflict of Interests}

The authors declare that they have no conflict of interests.

\section{Authors' Contribution}

All authors contributed equally to this work. 


\section{References}

[1] H. Madersbacher, "Neurogenic bladder dysfunction in patients with myelomeningocele," Current Opinion in Urology, vol. 12, no. 6, pp. 469-472, 2002.

[2] H. Schulte-Baukloh, T. Michael, B. Stürzebecher, and H. H. Knispel, "Botulinum-A toxin detrusor injection as a novel approach in the treatment of bladder spasticity in children with neurogenic bladder," European Urology, vol. 44, no. 1, pp. 139-143, 2003.

[3] G. Del Popolo, La Tossina Botulinica in Neurourologia, Somipar, 2001.

[4] A. Reitz, M. Stöhrer, G. Kramer et al., "European experience of 200 cases treated with botulinum-A toxin injections into the detrusor muscle for urinary incontinence due to neurogenic detrusor overactivity," European Urology, vol. 45, no. 4, pp. 510-515, 2004

[5] D. Sussman, V. Patel, G. Del Popolo, W. Lam, D. Globe, and P. Pommerville, "Treatment satisfaction and improvement in health-related quality of life with onabotulinumtoxinA in patients with urinary incontinence due to neurogenic detrusor overactivity," Neurourology and Urodynamics.

[6] K. F. Neel, M. Salem, and S. Soliman, "Total endoscopic management (TEM approach) of children with non-compliant neuropathic bladder: a preliminary report," Journal of Pediatric Urology, vol. 4, no. 2, pp. 124-126, 2008.

[7] E. J. McGuire, J. R. Woodside, T. A. Borden, and R. M. Weiss, "Prognostic value of urodynamic testing in myelodysplastic patients," Journal of Urology, vol. 126, no. 2, pp. 205-209, 1981.

[8] A. M. Houle, R. F. Gilmour, B. M. Churchill, M. Gaumond, and B. Bissonnette, "What volume can a child normally store in the bladder at a safe pressure?" Journal of Urology, vol. 149, no. 3, pp. 561-564, 1993

[9] A. Marte, M. Borrelli, M. D. Sabatino et al., "Effectiveness of botulinum-A toxin for the treatment of refractory overactive bladder in children," European Journal of Pediatric Surgery, vol. 20, no. 3, pp. 153-157, 2010.

[10] J. Blasi, E. R. Chapman, E. Link et al., "Botulinum neurotoxin A selectively cleaves the synaptic protein SNAP-25," Nature, vol. 365, no. 6442, pp. 160-163, 1993.

[11] O. Dolly, "Synaptic transmission: inhibition of neurotransmitter release by botulinum toxins," Headache, vol. 43, no. 1, pp. S16-S24, 2003.

[12] P. Denys, A. Even-Schneider, I. T. Escudie, D. B. Smail, N. Ayoub, and E. Chartier-Kastler, "Efficacy of botulinum toxin A for the treatment of detrusor hyperreflexia," Annales de Readaptation et de Medecine Physique, vol. 46, no. 6, pp. 326-328, 2003.

[13] T. Leippold, A. Reitz, and B. Schurch, "Botulinum toxin as a new therapy option for voiding disorders: current state of the art," European Urology, vol. 44, no. 2, pp. 165-174, 2003.

[14] B. Schurch, M. Stöhrer, G. Kramer, D. M. Schmid, G. Gaul, and D. Hauri, "Botulinum-A toxin for treating detrusor hyperreflexia in spinal cord injured patients: a new alternative to anticholinergic drugs? Preliminary results," Journal of Urology, vol. 164, no. 3, pp. 692-697, 2000.

[15] U. Mehnert, S. Boy, M. Schmid et al., "A morphological evaluation of botulinum neurotoxin A injections into the detrusor muscle using magnetic resonance imaging," World Journal of Urology, vol. 27, no. 3, pp. 397-403, 2009.

[16] H. Schulte-Baukloh, J. Herholz, H. Bigalke, K. Miller, and H. H. Knispel, "Results of a BoNT/An antibody study in children and adolescents after onabotulinumtoxin A (Botox) detrusor injection," Urologia Internationalis, vol. 87, no. 4, pp. 434-438, 2011.

[17] X. Gamé, P. Mouracade, E. Chartier-Kastler et al., "Botulinum toxin-A (Botox) intradetrusor injections in children with neurogenic detrusor overactivity/neurogenic overactive bladder: a systematic literature review," Journal of Pediatric Urology, vol. 5, no. 3, pp. 156-164, 2009.

[18] J. Grosse, G. Kramer, and M. Stöhrer, "Success of repeat detrusor injections of botulinum A toxin in patients with severe neurogenic detrusor overactivity and incontinence," European Urology, vol. 47, no. 5, pp. 653-659, 2005.

[19] B. Wefer, B. Ehlken, J. Bremer et al., "Treatment outcomes and resource use of patients with neurogenic detrusor overactivity receiving botulinum toxin A (BOTOX) therapy in Germany," World Journal of Urology, vol. 28, no. 3, pp. 385-390, 2010. 


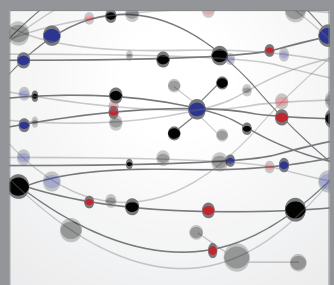

The Scientific World Journal
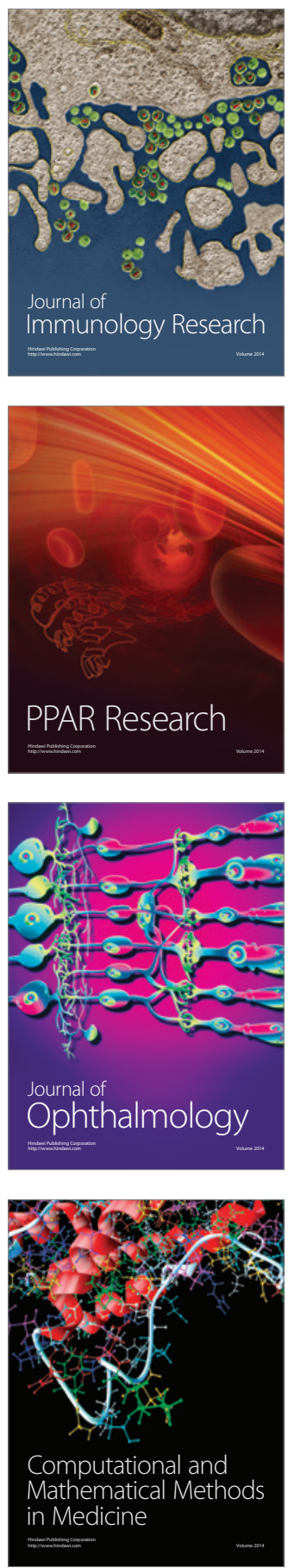

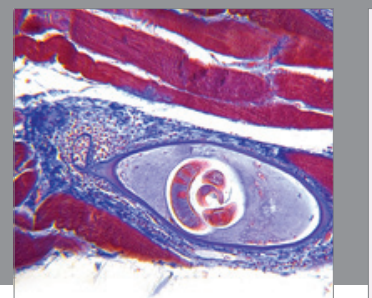

Gastroenterology

Research and Practice
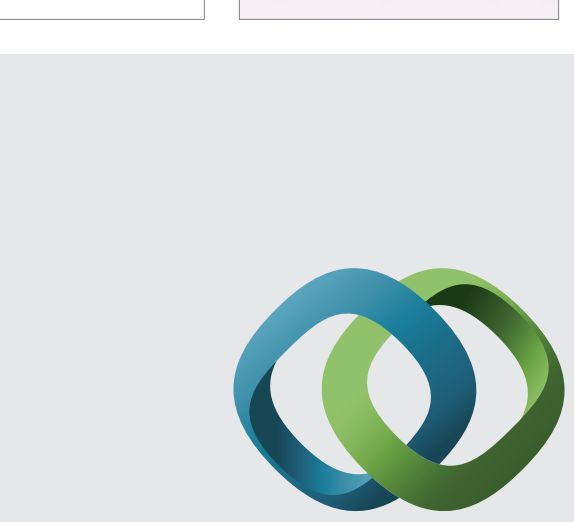

\section{Hindawi}

Submit your manuscripts at

http://www.hindawi.com
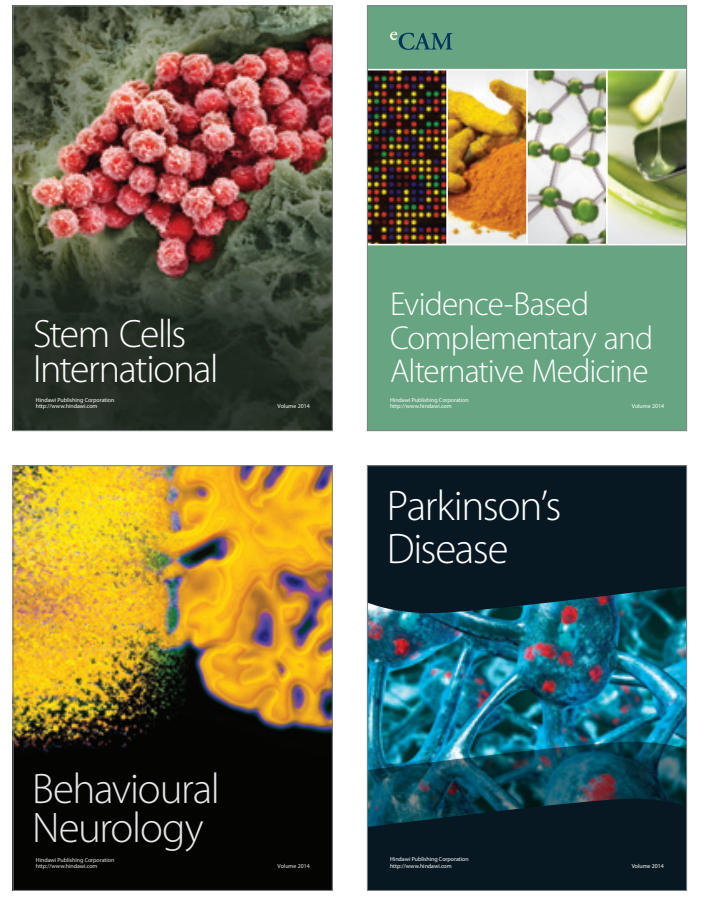
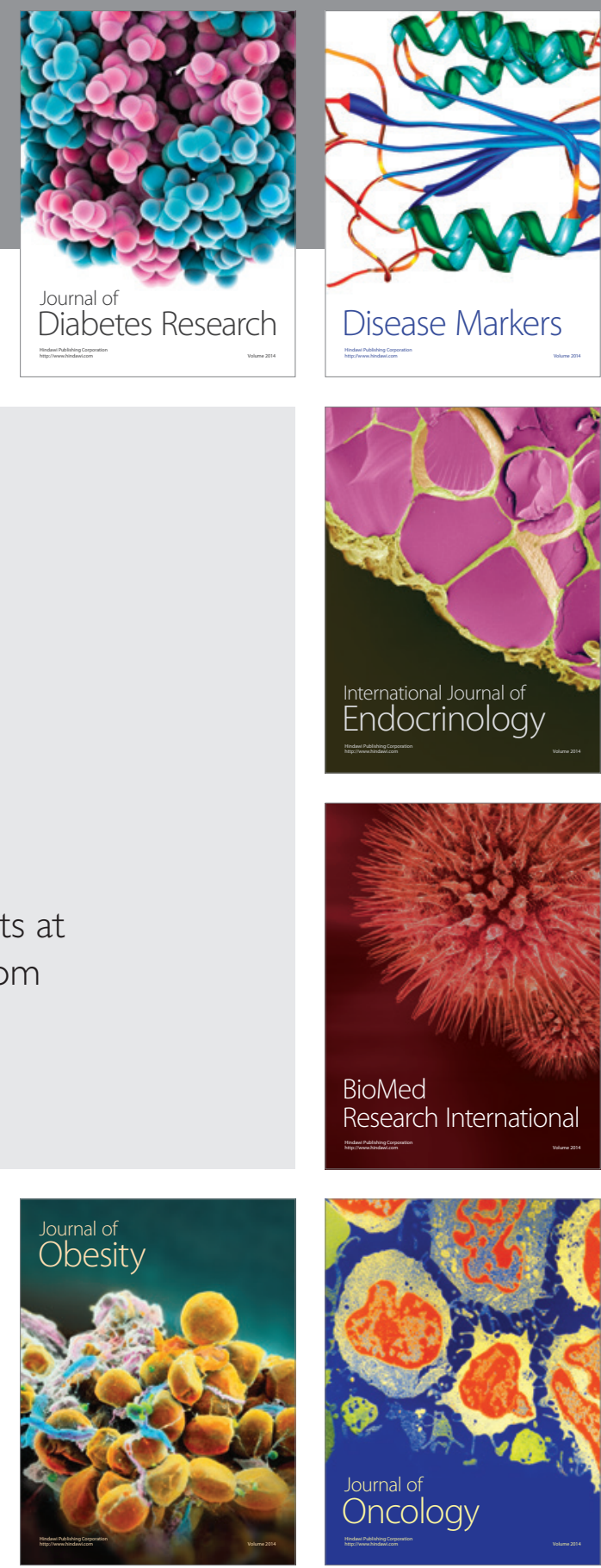

Disease Markers
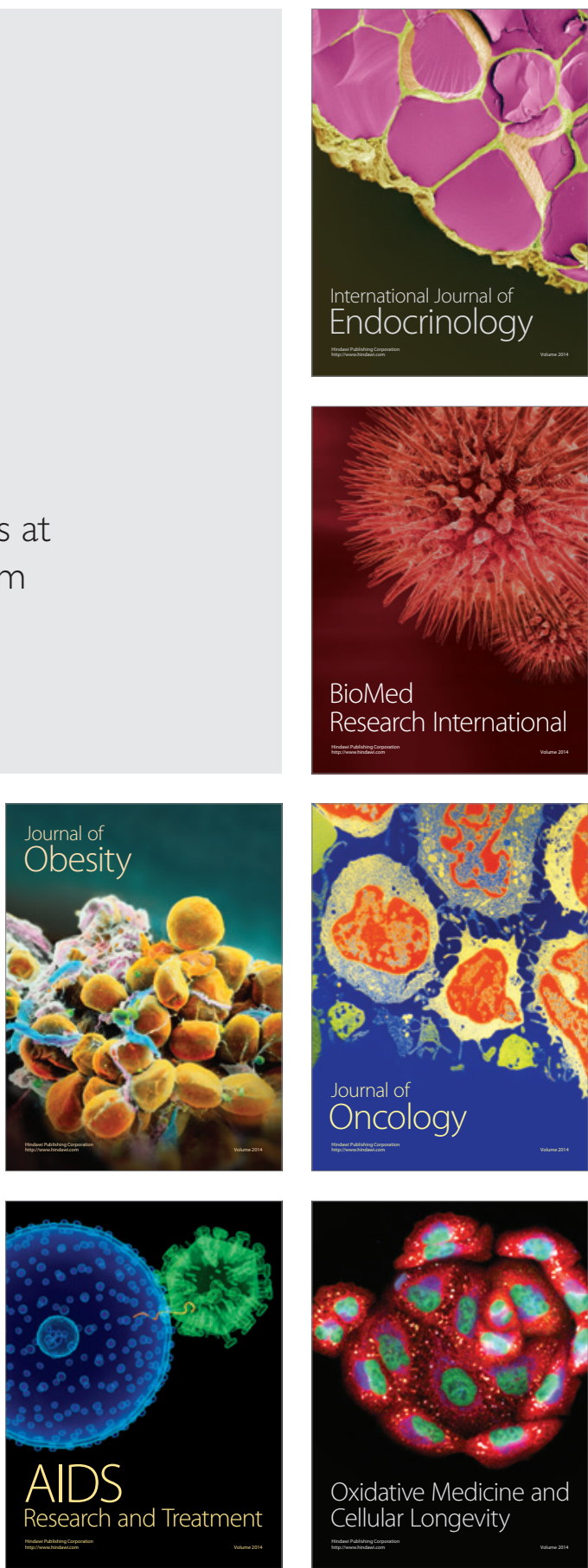